\title{
Sudden change in quantum and classical correlations and the Unruh effect
}

\author{
L. C. Céleri, ${ }^{1, *}$ A. G. S. Landulfo, ${ }^{2, \dagger}$ R. M. Serra, ${ }^{1, \ddagger}$ and G. E. A. Matsas ${ }^{2, \S}$ \\ ${ }^{1}$ Centro de Ciências Naturais e Humanas, Universidade Federal do ABC, Rua Santa Adélia 166, \\ 09210-170 Santo André, São Paulo, Brazil \\ ${ }^{2}$ Instituto de Física Teórica, Universidade Estadual Paulista, Rua Dr. Bento Teobaldo Ferraz, 271-Bl. II, \\ 01140-070 São Paulo, São Paulo, Brazil
}

(Received 23 March 2010; published 28 June 2010)

\begin{abstract}
We use the Unruh effect to analyze the dynamics of classical and quantum correlations for a two-qubit system when one of them is uniformly accelerated for a finite amount of proper time. We show that the quantum correlation is completely destroyed in the limit of infinite acceleration, while the classical one remains nonzero. In particular, we show that such correlations exhibit the so-called sudden-change behavior as a function of acceleration. Eventually, we discuss how our results can be interpreted when the system lies in the vicinity of the event horizon of a Schwarzschild black hole.
\end{abstract}

DOI: 10.1103/PhysRevA.81.062130 PACS number(s): 03.65.Ud, 03.30.+p, 03.67.-a, 04.62.+v

\section{INTRODUCTION}

What is quantum in a quantum correlated system? Until recently, the usual answer to this question was entanglement. However, as pointed out by Ollivier and Zurek [1] (see also Ref. [2]), separable states may still present quantum correlations. Recent results showing that almost all (separable) quantum states actually have such nonclassical correlations [3] and that they can improve some computational tasks in comparison to when classical states are used [4,5] reinforce the relevance of such an issue. As a result, the last few years have witnessed an increasing number of articles discussing the quantification of these correlations [1,2,6-8], their behavior under decoherence [9-12], and their relevance for quantum phase transitions [13-16].

Parallel to these developments, theoretical studies analyzing the role played by relativity in the behavior of quantum systems when (i) the relative motion and (ii) proper acceleration of the communicating partners is large have attracted much attention (see Ref. [17] for a recent review). This is not only interesting in its own right but may also have practical importance because of new trends of implementing quantum-information protocols at global scales through the use of satellite systems [18-22]. Concerning condition (i), the remarkable fact was shown in Ref. [23] that the von Neumann entropy associated with the reduced spin-density matrix of a single particle is not Lorentz invariant. This is so because, in general, the spin is Wigner rotated under Lorentz boosts in a direction which depends on the particle momentum, thus entangling both degrees of freedom. Similarly, it was shown that the entanglement for a two-particle spin system is not Lorentz invariant either [24]. The Lorentz invariance of the entanglement distillability of a bipartite mixed spin state was investigated in Ref. [25]. The degree of violation of the Clauser-Horne-Shimony-Holt inequality as seen by

\footnotetext{
*lucas.celeri@ufabc.edu.br

†landulfo@ift.unesp.br

${ }^{\ddagger}$ serra@ufabc.edu.br

§matsas@ift.unesp.br
}

different inertial observers for two entangled fermions was studied in Ref. [26] and for entangled photons in Ref. [27]. Concerning condition (ii), it was shown in Ref. [28] that uniformly accelerated observers see a degradation in the degree of entanglement between two bosonic modes in comparison to inertial observers. This fact can be explained through the well-known Unruh effect [29], which states that uniformly accelerated observers in Minkowski space-time associate a thermal bath of Rindler particles to the Minkowski vacuum (see, e.g., Ref. [30] for a review). The case of continuous variables was studied in Ref. [31], where it was shown that the entanglement is completely destroyed in the limit of infinite acceleration for scalar fields, in contrast to what was found for Dirac ones [32]. In a recent work, two of the present authors observed sudden death of entanglement in a two-qubit system when one of the partners is accelerated by some external agent [33]. A similar result for circularly moving qubits can be found in Ref. [34].

A common feature shared by the previous articles is that all of them consider some entanglement measure to quantify the quantum correlation contained in composite systems. Here we are interested in the dynamics of a more general class of (classical and quantum) correlations as given, for example, by the quantum discord [1]. For this purpose we consider a two-qubit system where one of the partners is uniformly accelerated for a finite amount of proper time. We verify that the quantum and classical correlations exhibit a suddenchange behavior [9] as a function of the noninertial qubit acceleration. In addition, we show that the quantum correlation is completely destroyed in the limit of infinite acceleration in contrast to the classical one. An analysis that complements ours can be found in Ref. [35], where the author studies the quantum discord between two free modes from the point of view of relatively accelerated observers. He finds that the quantum correlation between the modes is not entirely destroyed from the point of view of these observers, even in the limit of infinite acceleration. It is important to note that the setup in Ref. [35] differs from ours, where one of the qubits is uniformly accelerated and thus under the influence of some external force.

The article is organized as follows. In Sec. II, we introduce our two-qubit system. In Sec. III, we discuss some quantifiers 
of classical and quantum correlations. Section IV is dedicated to presenting our results on the behavior of the correlations, which are also discussed in terms of the Unruh effect. In Sec. V, we explain how our results can be straightforwardly applied when the qubits are in the vicinity of the event horizon of a black hole. Our final remarks are presented in Sec. VI. Throughout this article all logarithms are taken to base 2 and we adopt natural units: $\hbar=c=G=k_{B}=1$.

\section{ALICE-ROB PROBLEM}

In this section, we briefly introduce the qubit system considered here. (We refer to Ref. [33] for more details.)

\section{A. Qubit model}

Our qubits are modeled as two-level semiclassical detectors. The semiclassical character of the detector, usually named after Unruh-DeWitt, relies on the fact that, while it possesses a well-defined classical world line, its internal degrees of freedom are treated quantum mechanically [30]. The detector proper Hamiltonian is defined as

$$
H_{D}=\Omega D^{\dagger} D,
$$

where $\Omega>0$ is the detector energy gap and $D^{\dagger}, D$ are the transition operators for the qubit energy eigenstates: $D^{\dagger}|1\rangle=$ $D|0\rangle=0, D^{\dagger}|0\rangle=|1\rangle$, and $D|1\rangle=|0\rangle$. Here $|0\rangle$ and $|1\rangle$ represent the unexcited and excited qubit energy eigenstates, respectively.

We coupled the qubit to a massless scalar field operator $\phi(x)$ through the interaction Hamiltonian

$$
H_{I}=\epsilon(t) \int_{\Sigma_{t}} d^{3} \mathbf{x} \sqrt{-g} \phi(x)\left[\psi(\mathbf{x}) D+\psi^{*}(\mathbf{x}) D^{\dagger}\right],
$$

where $g=\operatorname{det}\left(g_{a b}\right), g_{a b}$ is the Minkowski space-time metric, and $\mathbf{x}$ are coordinates defined on the Cauchy surface $\Sigma_{t=\text { const }}$ associated with the suitable timelike isometries followed by the qubits (namely, the inertial and uniformly accelerated ones). The smooth compact support real-valued function $\epsilon$ is introduced to keep the detector switched on for a finite amount of proper time $\Delta$, which is always assumed to have a fixed nonzero value here, and $\psi$ is a smooth compact support complex-valued function modeling the fact that the detector only interacts with the field in a neighborhood of its world line. The total Hamiltonian can be cast as

$$
H=H_{0}+H_{I},
$$

where $H_{0}=H_{D}+H_{\mathrm{KG}}$ and $H_{\mathrm{KG}}$ is the free scalar field Hamiltonian. By using the interaction picture, the final state of the qubit-field system in first perturbation order is given by [36]

$$
\left|\Psi_{\infty}\right\rangle=\left[I+a^{\dagger}(\lambda) D-a(\bar{\lambda}) D^{\dagger}\right]\left|\Psi_{-\infty}\right\rangle,
$$

where $\left|\Psi_{-\infty}\right\rangle$ is the corresponding initial state, $\lambda=-K E f$, $f=\epsilon(t) \psi(\mathbf{x}) e^{-i \Omega t}, E$ is the difference between the advanced and retarded Green functions, $K$ is an operator that takes the positive frequency part of the solutions of the Klein-Gordon equation with respect to the timelike isometry, and $a(\bar{u})$ and $a^{\dagger}(u)$ are the annihilation and creation operators of $u$ modes, respectively. Equation (4) carries the physical message that the excitation and de-excitation of an Unruh-DeWitt detector following a timelike isometry is associated with the absorption and emission, respectively, of a particle as "naturally" defined by observers co-moving with the detector. Only processes where the detector flips once or none at all are considered here.

\section{B. Alice, Rob, and the Unruh effect}

Let us now consider two noninteracting qubits in Minkowski space-time. The one carried by Alice is kept inertial while the one carried by Rob has constant proper acceleration $a$ along the $x$ axis for the finite amount of proper time $\Delta$. The world line of Rob's qubit is given by

$$
t(\tau)=a^{-1} \sinh a \tau, \quad x(\tau)=a^{-1} \cosh a \tau,
$$

$y(\tau)=z(\tau)=0$, where $\tau$ is the qubit proper time and $(t, x, y, z)$ are the usual Cartesian coordinates of Minkowski space-time. This is meaningful as long as we consider spacelocalized qubits, which can be realized by choosing $\psi(\mathbf{x})=$ $(\kappa \sqrt{2 \pi})^{-3} \exp \left(-\mathbf{x}^{2} / 2 \kappa^{2}\right)$ with variance $\kappa=$ const $\ll \Omega^{-1}$.

The initial state describing the complete system composed of the two qubits and scalar field is denoted as

$$
\begin{aligned}
\left|\Psi_{0}^{A R \phi}\right\rangle & =\left|\Psi_{0}^{A R}\right\rangle \otimes\left|0_{M}\right\rangle \\
& =\frac{1}{\sqrt{2}}\left(\left|0_{A}\right\rangle \otimes\left|1_{R}\right\rangle-\left|1_{A}\right\rangle \otimes\left|0_{R}\right\rangle\right) \otimes\left|0_{M}\right\rangle,
\end{aligned}
$$

where $\left\{\left|0_{X}\right\rangle,\left|1_{X}\right\rangle\right\}$ is an orthonormal basis of the internal qubit space; $X=A$ and $R$ labels the Alice and Rob qubits, respectively; and $\left|0_{M}\right\rangle$ is the scalar field Minkowski vacuum, that is, the no-particle state as defined by inertial observers.

The free Hamiltonian for each qubit is given by Eq. (1) with the appropriate substitution $D \rightarrow A, R$. They are designed such that Alice's qubit remains always switched off while Rob's qubit is kept switched on during the nonzero time interval $\Delta$ along which it interacts with the field through the effective coupling constant $v^{2}$ [see Eq. (8)]. As a result, Rob's qubit interacts with the scalar field as ruled by Eq. (2) (with $D \rightarrow R$ and $t \rightarrow \tau$ ), while Alice's qubit interacts with the scalar field only indirectly. Then, the total Hamiltonian of the complete two-qubit system interacting with the field is given by

$$
H=H_{A}+H_{R}+H_{\mathrm{KG}}+H_{I} .
$$

Now, by using Eq. (4) to evolve our initial state (5) and tracing out the field degrees of freedom eventually, we obtain the final reduced density matrix [33]

$$
\rho_{\infty}^{A R}=\left[\begin{array}{cccc}
S_{2} & 0 & 0 & 0 \\
0 & S_{0} & -S_{0} & 0 \\
0 & -S_{0} & S_{0} & 0 \\
0 & 0 & 0 & S_{1}
\end{array}\right]
$$

associated with the two-qubit degrees of freedom in the basis

$$
\left\{\left|0_{A}\right\rangle \otimes\left|0_{R}\right\rangle,\left|1_{A}\right\rangle \otimes\left|0_{R}\right\rangle,\left|0_{A}\right\rangle \otimes\left|1_{R}\right\rangle,\left|1_{A}\right\rangle \otimes\left|1_{R}\right\rangle\right\},
$$


where

$$
\begin{aligned}
& S_{0}=\frac{1-q}{2(1-q)+v^{2}(1+q)}, \\
& S_{1}=\frac{v^{2} q}{2(1-q)+v^{2}(1+q)}, \\
& S_{2}=\frac{v^{2}}{2(1-q)+v^{2}(1+q)} .
\end{aligned}
$$

For the sake of convenience, we have defined the parametrized acceleration $q \equiv e^{-2 \pi \Omega / a}$, as well as the effective coupling

$$
v^{2} \equiv\|\lambda\|^{2}=\frac{\epsilon^{2} \Omega \Delta}{2 \pi} e^{-\Omega^{2} \kappa^{2}} .
$$

We note that a couple of necessary conditions for the relations above to be valid is that $\Omega^{-1} \ll \Delta$ and that $\epsilon$ be a slowly varying function of time when compared to the frequency $\Omega$.

For the asymptotic limit of infinite acceleration $(q \rightarrow 1)$, the final reduced density matrix of the Alice-Rob system turns out to be [see Eq. (7)]

$$
\left.\rho_{\infty}^{A R}\right|_{q \rightarrow 1}=\frac{1}{2}\left|0_{A} 0_{R}\right\rangle\left\langle 0_{A} 0_{R}\left|+\frac{1}{2}\right| 1_{A} 1_{R}\right\rangle\left\langle 1_{A} 1_{R}\right| .
$$

The asymptotic state (9) is a consequence of the assumption that the detector is allowed to flip only once or none at all [33] and the fact that in the infinite acceleration limit Rob's detector must necessarily flip; no flip is not an option in this case. Because each detector excitation and de-excitation in the usual inertial vacuum is necessarily associated with the emission of a Minkowski particle, inertial observers must discard data coming from experiments where two or more Minkowski particles are eventually left by using some postselection process. Accordingly, uniformly accelerated observers must also discard data associated with two or more detector transitions.

\section{QUANTUM AND CLASSICAL CORRELATIONS}

In classical information theory, the correlation between two random variables $A$ and $B$ is measured by the mutual information [37]

$$
I_{c}(A: B)=\mathcal{H}(A)+\mathcal{H}(B)-\mathcal{H}(A, B),
$$

where $\mathcal{H}(X)=-\sum_{x} p_{x} \log p_{x}$ is the usual Shannon entropy for the variable $X$ and $\mathcal{H}(X, Y)=-\sum_{x, y} p_{x, y} \log p_{x, y}$ is the corresponding joint entropy. Here $p_{x}$ is the probability of variable $X$ to assume the value $x$ and $p_{x, y} \equiv p(y \mid x) p(x)$, where $p(y \mid x)$ is the conditional probability of occurrence of $y$ when $x$ has already occurred. Accordingly, Eq. (10) can be straightforwardly extended for a bipartite quantum state described by the density matrix $\rho_{A B}$ as follows [38-40]:

$$
\mathcal{I}\left(\rho_{A: B}\right)=S\left(\rho_{A}\right)+S\left(\rho_{B}\right)-S\left(\rho_{A B}\right),
$$

where $S(\rho)=-\operatorname{Tr}(\rho \log \rho)$ is the von Neumann entropy, $\rho_{A}=$ $\operatorname{Tr}_{B}\left(\rho_{A B}\right)$ is the reduced density operator of the partition $A$, and accordingly for $\rho_{B}$. Equation (11) gives a measure of the total correlations (including the quantum and the classical ones) contained in a bipartite quantum system $[9,10,41,42]$. The classical part of correlation (11) can be expressed as being the "maximum classical mutual information" obtained by local measurements on both partitions of a composite state [6]:

$$
\mathcal{K}\left(\rho_{A B}\right)=\max _{\left\{\Pi_{i}^{(A)} \otimes \Pi_{j}^{(B)}\right\}}\left[I_{c}\left(\rho_{A: B}\right)\right] .
$$

Here $I_{c}\left(\rho_{A: B}\right)$ is given by Eq. (10) provided that $\mathcal{H}(X)$ is seen in this case as the entropy of the probability distribution of system $X$ resulting from a set of local projective measurements $\Pi_{i}^{(A)} \otimes \Pi_{j}^{(B)}$ on both subsystems $A$ and $B$. The maximization is taken over the set of all possible projective measurements.

Due to the distinct nature of both classical and quantum correlations, it is reasonable to assume that they add in a simple way $[9,10,41,43,44]$. Hence, we can define the quantum part of the total correlation as

$$
\mathcal{Q}\left(\rho_{A B}\right) \equiv \mathcal{I}\left(\rho_{A: B}\right)-\mathcal{K}\left(\rho_{A B}\right) .
$$

Note that we are using here the "two-side" correlation measure (in the sense that both subsystems are measured) [10], instead of the "one-side" correlation measure as assumed in the quantum discord [1]. This choice relies on the fact that the Alice-Rob problem considered in this article is not symmetric under the permutation of the subsystems, since Alice and Rob are inertial and noninertial, respectively.

For the sake of completeness, we compare the results obtained by means of Eq. (13) with those obtained through the quantum discord

$$
\mathcal{D}\left(\rho_{A B}\right) \equiv \mathcal{I}\left(\rho_{A: B}\right)-\max _{\left\{\Pi_{j}^{(B)}\right\}} \mathcal{J}\left(\rho_{A: B}\right) .
$$

Here

$$
\mathcal{J}\left(\rho_{A: B}\right)=S\left(\rho_{A}\right)-S_{\left\{\Pi_{j}^{(B)}\right\}}\left(\rho_{A \mid B}\right),
$$

where $S_{\left\{\Pi_{j}^{(B)}\right\}}\left(\rho_{A \mid B}\right)$ is the quantum extension of the classical conditional entropy $\mathcal{H}(A \mid B)$, which (in the quantum case) depends on the measurement choice. Thus, to compute the quantum discord, we have to maximize this quantity over the set $\left\{\Pi_{j}^{(B)}\right\}$ of all possible projective measurements on subsystem $B$. Of course, there is a related one-side measure of classical correlation given by $\mathcal{C}\left(\rho_{A B}\right) \equiv \mathcal{I}\left(\rho_{A: B}\right)-\mathcal{D}\left(\rho_{A B}\right)$.

\section{DYNAMICS OF CORRELATIONS AND THE UNRUH EFFECT}

Let us now proceed to analyze the behavior of both classical and quantum correlations given by Eqs. (12) and (13), respectively, assuming point detectors: $\kappa=0$. In Fig. 1, the quantum and classical correlations as well as the mutual information are plotted as functions of the parametrized acceleration $q$ for a fixed value of $v^{2}$ and time interval $\Delta$ along which the detector stays switched on. First, we note that for null acceleration the total correlation value given by the quantum mutual information differs from 2 , as would be expected for the singlet state (5). This is so because even inertial detectors have a nonzero probability of spontaneously decaying (along the nonzero time interval $\Delta$ ) with the emission of a Minkowski particle, which carries away some information. Such a process leads to a purity loss of the initial singlet state degradating the initial correlations. It is worthwhile to emphasize that the usual value of 2 for the quantum mutual 


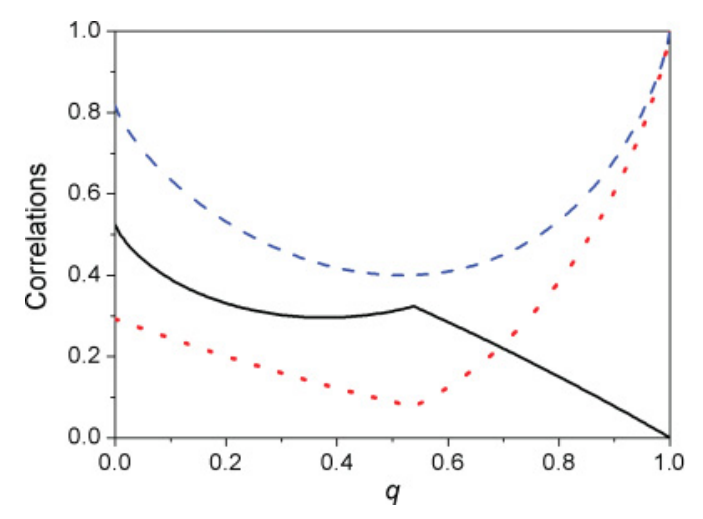

FIG. 1. (Color online) We plot the classical (dotted line) and quantum (solid line) correlations given by Eqs. (12) and (13), respectively, and the quantum mutual information (dashed line) given by Eq. (11) as functions of the parametrized acceleration $q$ considering the initial state (5). Here we set $v^{2}=0.4 \pi$.

information of the singlet state is obtained when the effective coupling constant $v^{2}$ vanishes, as can be seen from Fig. 2. In such a case there is no interaction between Rob's qubit and the scalar field. Figure 1 shows that the two-qubit system is still left with classical correlations in the limit of infinite acceleration $(q \rightarrow 1)$, while the quantum correlation vanishes. This fact is in agreement with Eq. (9) and, as mentioned before, this is a consequence of our detector model. From the point of view of uniformly accelerated observers, the loss of quantum correlation is due to the interaction of Rob's qubit with the Unruh thermal bath of Rindler particles that they experience when the field is in the Minkowski vacuum. We recall that the Unruh temperature experienced by the noninertial qubit is proportional to its proper acceleration. Now from the inertial observers' perspective, the quantum correlation is carried away by the scalar radiation emitted by the accelerating qubit when it suffers a transition. Another interesting result revealed by Fig. 1 is the fact that the dynamics of both classical and quantum correlations cannot be described by a smooth function of acceleration. Extensive numerical analyses indicate that this sudden change does not depend on the considered initial state. (The term "sudden change" was coined in Ref. [9]

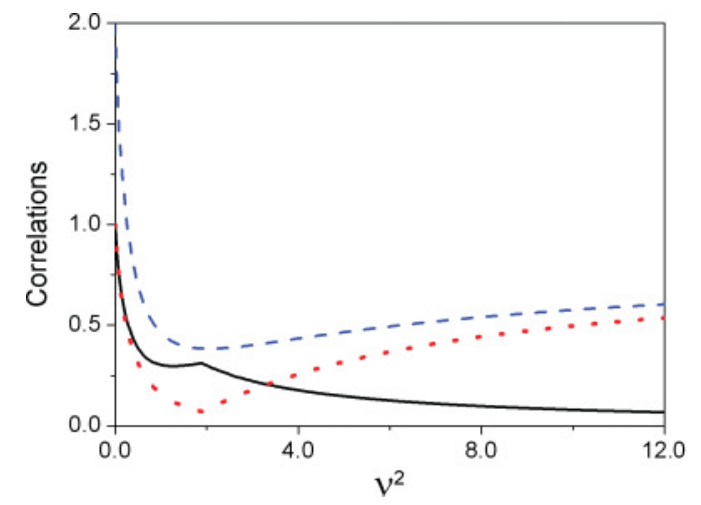

FIG. 2. (Color online) We plot the same correlations as in Fig. 1 with respect to $v^{2}$ assuming the initial state (5) and $q=0.4$. The quantum mutual information (11) is given by the dashed line and the classical and quantum correlations (12) and (13) are given by the dotted and solid lines, respectively.

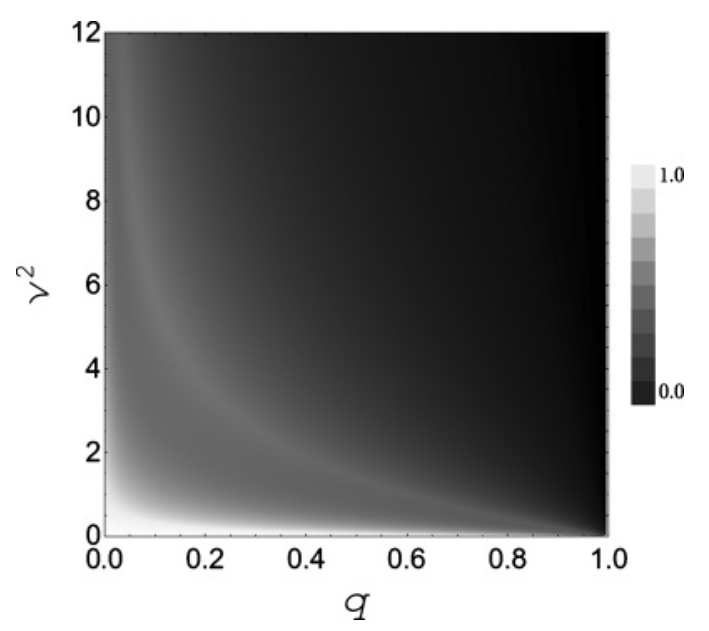

FIG. 3. Density plot of quantum correlation given in Eq. (13) as a function of $v^{2}$ and $q$, considering the initial state (5).

and experimentally observed in Ref. [45].) For larger values of $v^{2}$, the sudden-change point moves to the left on the $q$ axis. A similar behavior is observed when we plot the same correlations as functions of $v^{2}$ for a fixed value of acceleration, as seen in Fig. 2. The larger the acceleration, the closer to the origin the sudden-change point approaches. In Fig. 3, we show how the sudden-change point depends on the parameters $q$ and $v^{2}$. It becomes clear that the quantum correlation vanishes for every $v^{2}$ value provided that the acceleration is arbitrarily large. Figures 4 and 5 show the classical correlation (12) and quantum mutual information (11), respectively. It is clear that for arbitrarily large accelerations all correlations left in the two-qubit system have a classical rather than a quantum nature for all $v^{2}$ values.

As stated in Sec. I, there is no a priori reason to believe that the two quantifiers of quantum correlation, given by Eqs. (13) and (14), should lead to the same results for a system that is not symmetrical under permutation of its parts. Indeed, in our case they are shown to be distinct although close to each other (see Fig. 6).

An interesting fact that can also be seen from Fig. 6 is that both quantifiers appear to give the same value for the

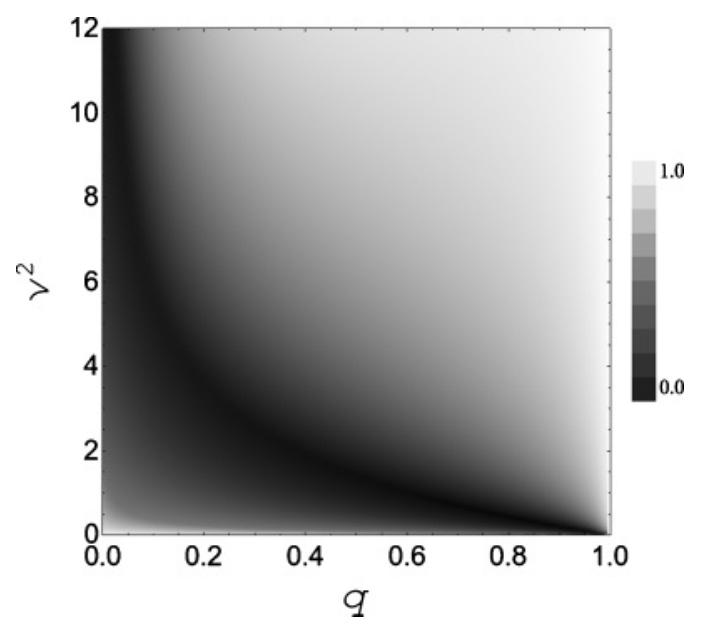

FIG. 4. Density plot of classical correlation given in Eq. (12) as a function of $v^{2}$ and $q$, considering the initial state (5). 


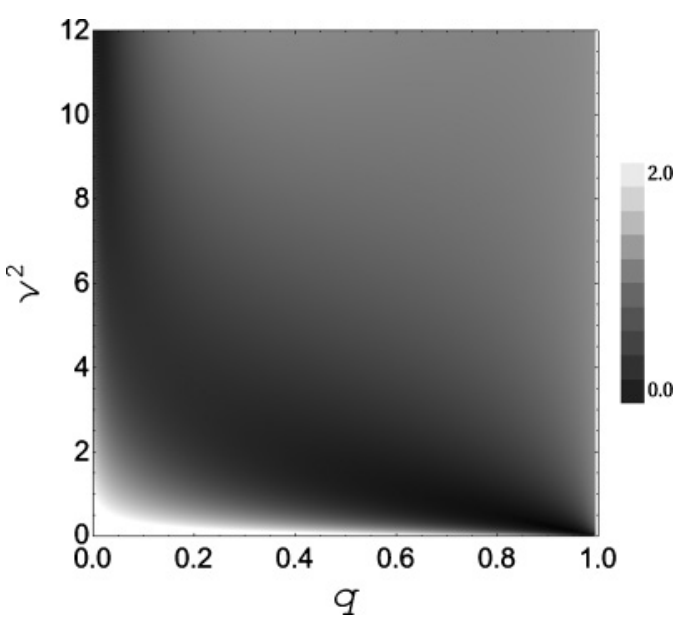

FIG. 5. Density plot of the quantum mutual information given in Eq. (11) as a function of $v^{2}$ and $q$, considering the initial state (5).

sudden-change point $q_{\mathrm{sc}}$. This allows us to derive in the present case an approximate analytical expression for $q_{\mathrm{sc}}$ as follows. In a recent article [12], it was shown that for all bipartite density operators of the form

$$
\rho_{A B}=\left[\begin{array}{cccc}
\rho_{11} & 0 & 0 & \rho_{14} \\
0 & \rho_{22} & \rho_{23} & 0 \\
0 & \rho_{23} & \rho_{22} & 0 \\
\rho_{14} & 0 & 0 & \rho_{33}
\end{array}\right],
$$

the quantum discord (14) is given by

$$
\mathcal{D}=\min \left\{D_{1}, D_{2}\right\} \quad\left(D_{1}, D_{2} \geqslant 0\right),
$$

where

$$
\begin{aligned}
D_{1}= & S\left(\rho_{A}\right)-S\left(\rho_{A B}\right)-\rho_{11} \log \left[\frac{\rho_{11}}{\rho_{11}+\rho_{22}}\right] \\
& -\rho_{22} \log \left[\frac{\rho_{22}^{2}}{\left(\rho_{11}+\rho_{22}\right)\left(\rho_{33}+\rho_{22}\right)}\right] \\
& -\rho_{33} \log \left[\frac{\rho_{33}}{\rho_{33}+\rho_{22}}\right],
\end{aligned}
$$

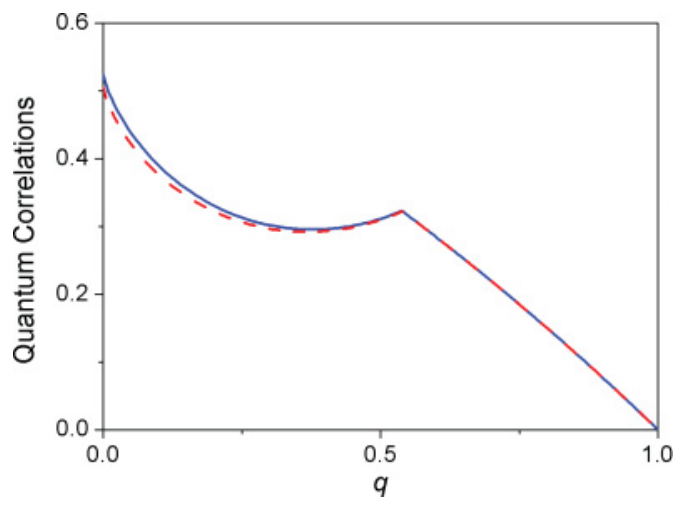

FIG. 6. (Color online) Plot of quantum correlations as functions of the parametrized acceleration $q$, setting $v^{2}=0.4 \pi$. The symmetrical measure (13) is shown by the solid line, while the quantum discord (14) is given by the dashed line (for measurements performed by either Alice or Rob).

$$
\begin{aligned}
D_{2}= & S\left(\rho_{A}\right)-S\left(\rho_{A B}\right)-\frac{1}{2}(1+\Gamma) \log \left[\frac{1}{2}(1+\Gamma)\right] \\
& -\frac{1}{2}(1-\Gamma) \log \left[\frac{1}{2}(1-\Gamma)\right],
\end{aligned}
$$

and $\Gamma^{2} \equiv\left(\rho_{11}-\rho_{33}\right)^{2}+4\left|\rho_{23}+\rho_{14}\right|^{2}$. By using the density operator $\rho_{\infty}^{A R}$ of Eq. (7) in Eq. (15), we obtain

$$
\begin{gathered}
D_{1}=2 S_{0}, \\
D_{2}=S\left(\rho_{A}\right)-S\left(\rho_{A B}\right)-\frac{1}{2} \log \left[\frac{1}{4}\left(1-\Gamma^{2}\right)\right] \\
+\frac{1}{2} \Gamma \log \left[\frac{1-\Gamma}{1+\Gamma}\right]
\end{gathered}
$$

with $\Gamma^{2}=\left(S_{2}-S_{1}\right)^{2}+4 S_{0}^{2}$. Then we see from Eq. (16) that the sudden-change point is obtained by solving

$$
D_{1}=D_{2}
$$

which turns out to be a transcendental equation for $q$ and $v^{2}$. For the case plotted in Fig. 1, Eq. (19) is satisfied for $q=q_{\mathrm{sc}} \approx$ 0.53925 , which is in agreement with the numerical analysis presented in the last section. An approximate semianalytical expression for $q_{\mathrm{sc}}$ can be found as follows. First, we find numerically all pairs $\left(q, v^{2}\right)$ which satisfy Eq. (19). Next, an exponential fitting

$$
q_{\mathrm{sc}}=\exp \left[a+b v^{2}+c v^{4}\right]
$$

is performed on these solutions with parameters

$$
a=0.00054, \quad b=-0.51488, \quad c=0.01959 .
$$

As we can see from Fig. 7, Eq. (20) is in very good agreement with the solutions of Eq. (19) for the range of parameters considered here. Figure 7 also shows the acceleration values

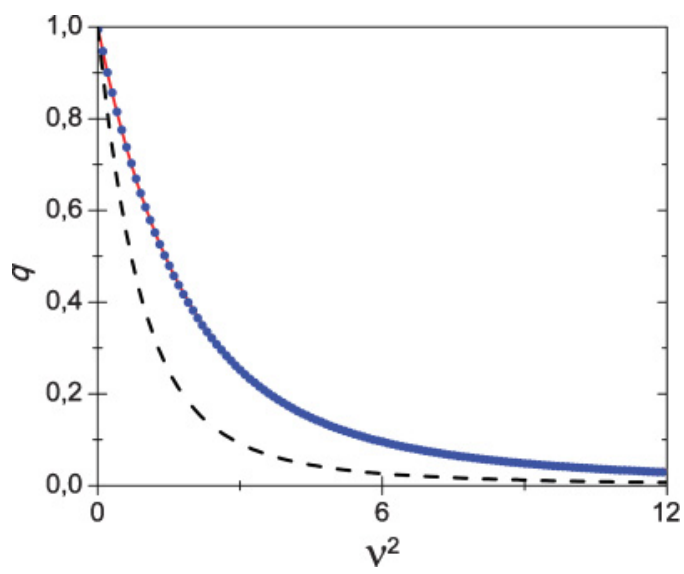

FIG. 7. (Color online) The dotted line shows the plot of the numerical solutions of Eq. (19). The solid line is the exponential fitting of these solutions. The dashed line is the plot of Eq. (21), showing the values of $q$ and $v^{2}$ for which the entanglement sudden death occurs. 


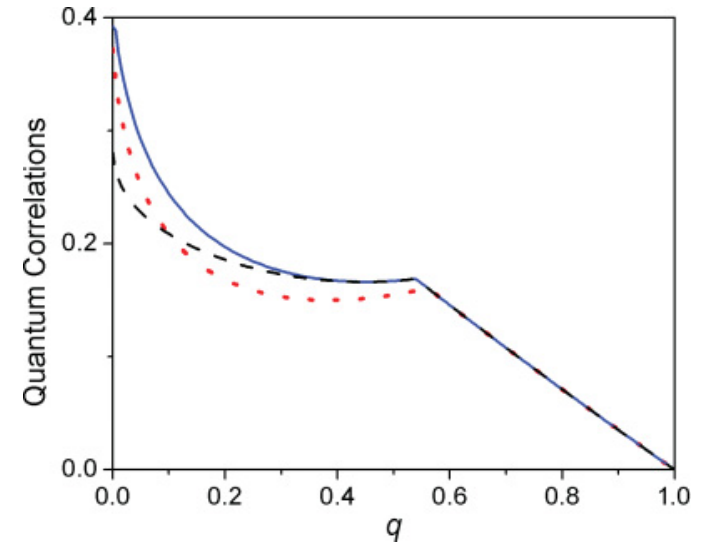

FIG. 8. (Color online) Plot of quantum correlations as a function of the parametrized acceleration $q$, setting $v^{2}=0.4 \pi$. The symmetrical measure (13) is shown by the solid line, while the quantum discord (14) is given by the dotted line (for a measurement performed by Rob) and by the dashed line (for a measurement performed by Alice).

$q_{\text {sd }}$ for which the entanglement sudden death [46] occurs, as given in Ref. [33]:

$$
q_{\mathrm{sd}}=\left(v^{2} / 2+\sqrt{1+v^{4} / 4}\right)^{-2},
$$

which suggests that the entanglement sudden death and the quantum correlation sudden change are uncorrelated. Furthermore, we note that the quantum correlation as defined by Eqs. (13) and (14) is still present after the vanishing of the entanglement.

Now, let us wonder what happens if we consider a nonsymmetrical initial state

$$
\left|\Psi_{0}^{\prime A R \phi}\right\rangle=\left(\alpha\left|0_{A}\right\rangle \otimes\left|1_{R}\right\rangle-\beta\left|1_{A}\right\rangle \otimes\left|0_{R}\right\rangle\right) \otimes\left|0_{M}\right\rangle
$$

under permutation of the $A$ and $R$ qubits, where $|\alpha|^{2}+$ $|\beta|^{2}=1$ rather than the symmetrical one (5). In Fig. 8, we plot the quantum discord (14) and the two-side quantum correlation (13) assuming the state (22) with $\alpha=0.3$. As we can see, the quantum discord for the two partners, Alice (inertial) and Rob (noninertial), differs from the two-side measure [Eq. (13)] and from each other, leading to distinct sudden-change points for the two observers. In such situations, using symmetrical correlation measures seems to be more suitable. For the cases considered in this article, the sudden changes associated with (i) the quantum discord as computed by the inertial observer and (ii) the two-side measurement always seem to agree with each other. However, Eq. (19) is not valid anymore due to the fact that the density operator for the Alice-Rob system is not in the form given by Eq. (15). Figure 9 shows a plot of Eq. (13) for the initial state (22), where we can see that the sudden-change point does not seem to vary with $\alpha$ (and $\beta$ ). Moreover, this critical point always exists except for the separable pure states, where $\alpha=0$ or 1 . The (omitted) plot for the quantum discord as measured by the inertial observer $A$ leads to a similar behavior with agreement on the sudden-change point.

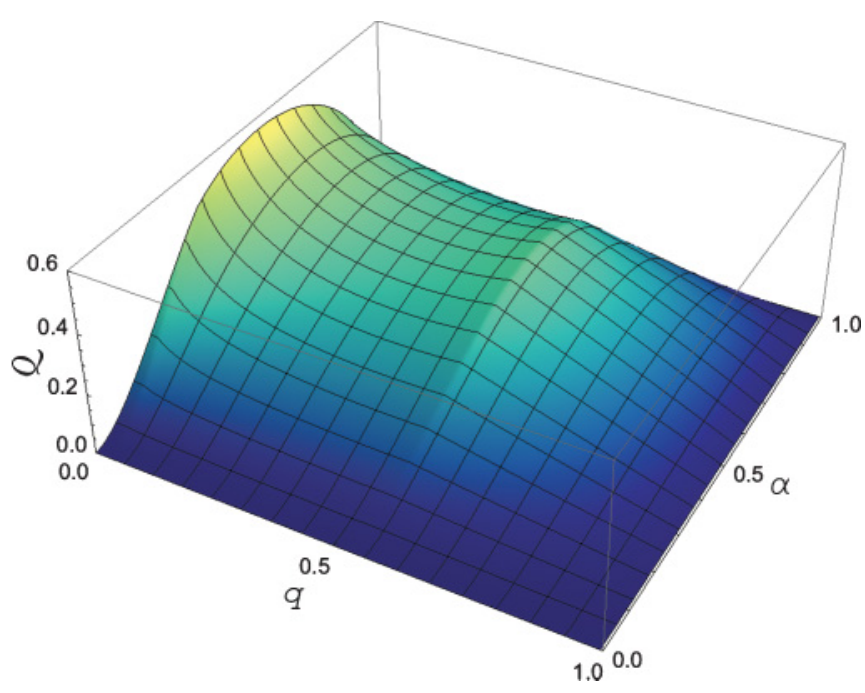

FIG. 9. (Color online) The quantum correlation given in Eq. (13) is plotted as a function of $\alpha$ and $q$ setting $v^{2}=0.4 \pi$ for the initial state (22). We note that it exhibits a sudden change across the $q \approx 0.54$ line.

\section{CLASSICAL AND QUANTUM CORRELATIONS IN THE VICINITY OF A BLACK HOLE}

Let us now explain how our previous results translate when the two qubits are very close to a Schwarzschild (i.e., rotationless and chargeless) black hole. First, let us recall that in this case there is a static Killing field $\chi$ which is timelike in regions I and II of the extended Schwarzschild space-time (see Fig. 10). The Hartle-Hawking vacuum is the unique nonsingular (Hadamard) state, which is invariant under the isometries generated by $\chi$ [47-51].

By a similar analysis used to derive the Unruh effect, it can be shown that the Hartle-Hawking vacuum $\left|0_{\mathrm{HH}}\right\rangle$ is a thermal state with respect to the time translation generated by $\chi$ when restricted to region I. This should be identified with the so-called Hawking radiation as seen by static observers lying outside the black hole. The corresponding reduced density matrix can be cast as $[47,50]$

$$
\rho_{\mathrm{HH}}=\prod_{i}\left(C_{i} \sum_{n_{i \mathrm{I}}} e^{-2 \pi n_{i \mathrm{I}} \omega_{i} / \kappa}\left|n_{i \mathrm{I}}\right\rangle\left\langle n_{i \mathrm{I}}\right|\right),
$$

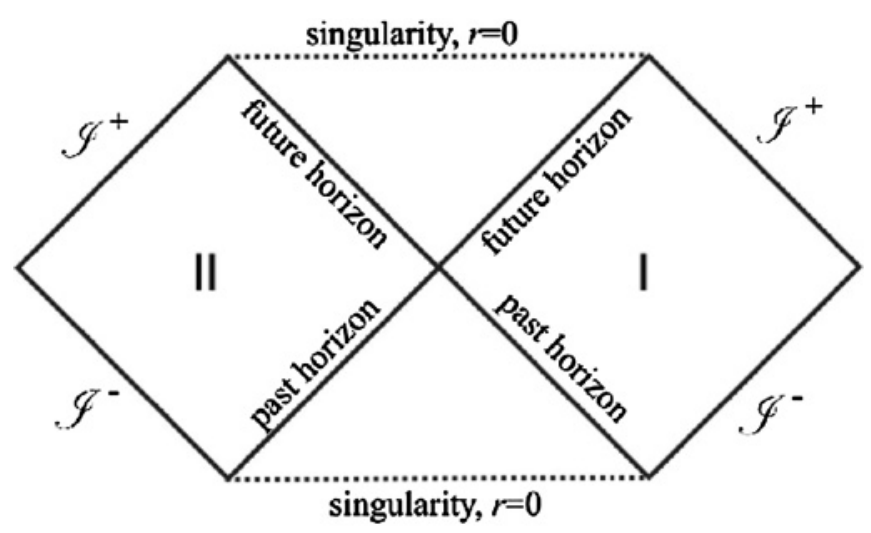

FIG. 10. Extended Schwarzschild space-time. 
where $\kappa=1 / 4 M$ is the surface gravity, $M$ is the black hole mass, $C_{i}=\left(1-e^{-2 \pi \omega_{i} / \kappa}\right)^{\frac{1}{2}}$, and $\left|n_{i \mathrm{I}}\right\rangle$ are states with $n$ particles in the modes $f_{i}^{\mathrm{I}}$. Here $f_{i}^{\mathrm{I}}$ and $f_{i}^{\mathrm{I} *}$ consist of a complete set of orthonormal $\chi$ positive-frequency solutions of the Klein-Gordon equation in region I and vanish in region II. We emphasize that, as a consequence both (i) uniformly accelerated observers in Minkowski vacuum $\left|0_{M}\right\rangle$ and (ii) static observers outside the black hole (who also have constant proper acceleration) in the Hartle-Hawking vacuum $\left|0_{\mathrm{HH}}\right\rangle$ experience a thermal bath of all particles. In contrast, free-falling observers in Minkowski space-time and in the vicinity of the black hole with the corresponding $\left|0_{M}\right\rangle$ and $\left|0_{\mathrm{HH}}\right\rangle$ vacua, respectively, see no particles at all [29].

Now, we determine the precise setup for Alice and Rob which allows the translation of our previous results for the black hole case. For this purpose, let us consider for the sake of simplicity the line element of a two-dimensional Schwarzschild black hole:

$$
d s^{2}=-(1-2 M / r) d t^{2}+(1-2 M / r)^{-1} d r^{2},
$$

where $(t, r)$ are the usual static Schwarzschild coordinates. By making the change of variables $r \rightarrow \rho(r)=\sqrt{8 M(r-2 M)}$, we see that very close to the horizon, $r \approx 2 M$, the metric takes the form

$$
d s^{2}=-(\rho / 4 M)^{2} d t^{2}+d \rho^{2} .
$$

This is the line element of the Rindler wedge provided that $0 \leqslant \rho<\infty$ and $-\infty<t<\infty$. The Rindler wedge is the section of the Minkowski space-time which is covered by uniformly accelerated observers, who see the Unruh thermal bath when the field state is in the Minkowski vacuum. Then, very near the event horizon, the Schwarzschild and Rindler space-times resemble each other. The fact that they differ asymptotically is not important provided that the qubits are localized near the horizon. As a matter of fact, this conclusion holds exactly the same for four-dimensional physical black holes. The "large" proper accelerations $a$ experienced by static observers near the black hole horizon are associated with small time scales in comparison with $r_{H}=2 M$, making any curvature effects negligible for our purposes. The local temperature measured by the static observers is given by $T=\kappa / 2 \pi V$, where $V=[-g(\chi, \chi)]^{1 / 2}$ is the redshift factor and the surface gravity can be cast as $\kappa=\lim _{\text {horizon }}(V a)$. Then, the temperature experienced by static observers very close to the horizon in the Hartle-Hawking vacuum is $T=a / 2 \pi$. This is analogous to the Unruh temperature for observers with constant proper acceleration $a$ in Minkowski vacuum.

As a result, all the conclusions of the previous sections continue to be valid in the vicinity of a Schwarzschild black hole with Hawking radiation provided that Alice is free falling and Rob is static with the same proper acceleration $a$ as if it were in Minkowski space-time. In particular, entanglement sudden death would be seen at finite acceleration $a_{\text {sd }}$ [33], while the quantum correlation would be completely destroyed only for $a \rightarrow \infty$ (i.e., when Rob is arbitrarily close to the black hole horizon). The sudden-change phe- nomenon would be observed accordingly at the same $q=q_{\mathrm{sc}}$ values.

\section{FINAL REMARKS}

Here, we have analyzed the behavior of quantum and classical correlations for a two-qubit system where one of them is uniformly accelerated while the other one is inertial. We have shown that the quantum correlation is degraded by the presence of acceleration. From the point of view of inertial observers, uniformly accelerated qubits have a nonzero probability of exciting with the emission of a Minkowski particle. This is possible because the external accelerating agent provides the necessary energy. Now, owing to the Unruh effect, coaccelerated observers with the qubit experience the Minkowski vacuum as a thermal bath (of Rindler particles). From their point of view, the qubit is in contact with the Unruh thermal bath and the excitation is because of the absorption of a particle from this hot reservoir. Clearly the physical observables encoded in the correlations do not depend on the particular observer description. In the limit of infinite acceleration, the Unruh thermal bath has an arbitrarily large temperature and it is natural to expect that quantum correlations be completely destroyed; however, classical correlations are still left [see Eq. (9)]. Another interesting point is that the behavior of both classical and quantum correlations cannot be described by smooth functions of the acceleration. They present a sudden change for a critical value of $q=q_{\mathrm{sc}}$. By comparing the quantum discord with a symmetrical quantifier for the quantum correlation, Eq. (13), we found that, although both measures are not exactly the same, they are very close to each other when we assume a symmetrical initial singlet state. This fact has allowed us to find an analytical fitting for $q_{\mathrm{sc}}$ in terms of $v^{2}$. Our results indicate that the correlation sudden-change and entanglement sudden-death points are uncorrelated in this scenario. Finally, by considering a nonsymmetrical rather than symmetrical initial state, we found that the quantum discord and the two-side measure for the quantum correlation become quite distinct. Also, the quantum discord as computed by the inertial $A$ and noninertial $R$ observers are distinct, leading to different sudden-change points $q_{\mathrm{sc}}$. This result suggests that symmetrical measures should be more suitable to be used to evaluate the correlations among the parties when the experimentalists have distinct inertial features as it would be the case when, for example, one of them is free falling and the other one is static in a gravitational field. Eventually the precise prescription of how our results can be translated when Alice is free falling and Rob is static in the vicinity of a Schwarzschild black hole is given.

\section{ACKNOWLEDGMENTS}

L.C. and A.L. acknowledge full financial support from Fundação Universidade Federal do ABC and Fundação de Amparo à Pesquisa do Estado de São Paulo (FAPESP), respectively. R.S. is grateful to Conselho Nacional de Desenvolvimento Científico e Tecnológico (CNPq), Instituto Nacional de Ciência e Tecnologia em Informação Quântica, and FAPESP for partial support. G.M. was partially supported by $\mathrm{CNPq}$ and FAPESP. 
[1] H. Ollivier and W. H. Zurek, Phys. Rev. Lett. 88, 017901 (2001).

[2] L. Henderson and V. Vedral, J. Phys. A: Math. Gen. 34, 6899 (2001); V. Vedral, Phys. Rev. Lett. 90, 050401 (2003).

[3] A. Ferraro, L. Aolita, D. Cavalcanti, F. M. Cucchietti, and A. Acín, Phys. Rev. A 81, 052318 (2010).

[4] A. Datta, A. Shaji, and C. M. Caves, Phys. Rev. Lett. 100, 050502 (2008).

[5] B. P. Lanyon, M. Barbieri, M. P. Almeida, and A. G. White, Phys. Rev. Lett. 101, 200501 (2008).

[6] B. M. Terhal, M. Horodecki, D. Leung, and D. P. DiVincenzo, J. Math. Phys. (NY) 43, 4286 (2002); D. P. DiVincenzo, M. Horodecki, D. W. Leung, J. A. Smolin, and B. M. Terhal, Phys. Rev. Lett. 92, 067902 (2004).

[7] S. Luo, Phys. Rev. A 77, 022301 (2008); J. Maziero, L. C. Celeri, and R. M. Serra, e-print arXiv:1004.2082.

[8] J. Oppenheim, M. Horodecki, P. Horodecki, and R. Horodecki, Phys. Rev. Lett. 89, 180402 (2002).

[9] J. Maziero, L. C. Celeri, R. M. Serra, and V. Vedral, Phys. Rev. A 80, 044102 (2009).

[10] J. Maziero, T. Werlang, F. F. Fanchini, L. C. Celeri, and R. M. Serra, Phys. Rev. A 81, 022116 (2010).

[11] T. Werlang, S. Souza, F. F. Fanchini, and C. J. Villas Boas, Phys. Rev. A 80, 024103 (2009).

[12] F. F. Fanchini, T. Werlang, C. A. Brasil, L. G. E. Arruda, and A. O. Caldeira, Phys. Rev. A 81, 052107 (2010); D. O. SoaresPinto, L. C. Celeri, R. Auccaise, F. F. Fanchini, E. R. de Azevedo, J. Maziero, T. J. Bonagamba, and R. M. Serra, ibid. 81, 062118 (2010).

[13] R. Dillenschneider, Phys. Rev. B 78, 224413 (2008).

[14] M. S. Sarandy, Phys. Rev. A 80, 022108 (2009).

[15] T. Werlang and G. Rigolin, Phys. Rev. A 81, 044101 (2010).

[16] J. Maziero, H. C. Guzman, L. C. Celeri, M. S. Sarandy, and R. M. Serra, e-print arXiv:1002.3906.

[17] A. Peres and D. R. Terno, Rev. Mod. Phys. 76, 93 (2004).

[18] M. Aspelmeyer et al., Science 301, 621 (2003).

[19] C.-Z. Peng et al., Phys. Rev. Lett. 94, 150501 (2005).

[20] R. Ursin et al., Nature Phys. 3, 481 (2007).

[21] R. Ursin et al., Proc. Int. Astronaut. Congr. A2.1.3 (2008).

[22] A. Fedrizzi et al., Nature Phys. 5, 389 (2009).

[23] A. Peres, P. F. Scudo, and D. R. Terno, Phys. Rev. Lett. 88, 230402 (2002).

[24] R. M. Gingrich and C. Adami, Phys. Rev. Lett. 89, 270402 (2002).

[25] L. Lamata, M. A. Martin-Delgado, and E. Solano, Phys. Rev. Lett. 97, 250502 (2006).

[26] A. G. S. Landulfo and G. E. A. Matsas, Phys. Rev. A 79, 044103 (2009).
[27] A. G. S. Landulfo, G. E. A. Matsas, and A. C. Torres, Phys. Rev. A 81, 044103 (2010).

[28] I. Fuentes-Schuller and R. B. Mann, Phys. Rev. Lett. 95, 120404 (2005).

[29] W. G. Unruh, Phys. Rev. D 14, 870 (1976).

[30] L. C. B. Crispino, A. Higuchi, and G. E. A. Matsas, Rev. Mod. Phys. 80, 787 (2008).

[31] G. Adesso, I. Fuentes-Schuller, and M. Ericsson, Phys. Rev. A 76, 062112 (2007).

[32] P. M. Alsing, I. Fuentes-Schuller, R. B. Mann, and T. E. Tessier, Phys. Rev. A 74, 032326 (2006).

[33] A. G. S. Landulfo and G. E. A. Matsas, Phys. Rev. A 80, 032315 (2009).

[34] J. Doukas and B. Carson, e-print arXiv:1003.2201v2.

[35] A. Datta, Phys. Rev. A 80, 052304 (2009).

[36] W. G. Unruh and R. M. Wald, Phys. Rev. D 29, 1047 (1984).

[37] T. M. Cover and J. A. Thomas, Elements of Information Theory (Wiley Interscience, New Jersey, 2006).

[38] M. A. Nielsen and I. L. Chuang, Quantum Computation and Quantum Information (Cambridge University, Cambridge, UK, 2000).

[39] G. Benenti, G. Casati, and G. Strini, Principles of Quantum Computation and Information, Vol. 2 (World Scientific, Singapore, 2007).

[40] V. Vedral, Introduction to Quantum Information Science (Oxford University, New York, 2007).

[41] B. Groisman, S. Popescu, and A. Winter, Phys. Rev. A 72, 032317 (2005).

[42] B. Schumacher and M. D. Westmoreland, Phys. Rev. A 74, 042305 (2006).

[43] J. Oppenheim, M. Horodecki, P. Horodecki, and R. Horodecki, Phys. Rev. Lett. 89, 180402 (2002).

[44] D. Yang, M. Horodecki, and Z. D. Wang, Phys. Rev. Lett. 101, 140501 (2008).

[45] J.-S. Xu, X.-Y. Xu, C.-F. Li, C.-J. Zhang, X.-B. Zou, and G.-C. Guo, Nature Commun. 1, 7 (2010).

[46] T. Yu and J. H. Eberly, Phys. Rev. Lett. 93, 140404 (2004)

[47] R. M. Wald, Quantum Field Theory in Curved Spacetimes and Black Hole Thermodynamics (University of Chicago, Chicago, 1994).

[48] B. S. Kay and R. M. Wald, Phys. Rep. 207, 49 (1991).

[49] J. B. Hartle and S. W. Hawking, Phys. Rev. D 13, 2188 (1976).

[50] W. Israel, Phys. Lett. A 57, 107 (1976).

[51] B. S. Kay, Commun. Math. Phys. 100, 57 (1985). 